\title{
Papilledema in the Setting of X-Linked Hypophosphatemic Rickets with Craniosynostosis
}

\author{
Lora R. Dagi Glass $^{a} \quad$ Teodoro Forcht Dagi $^{c} \quad$ Linda R. Dagi $^{b}$ \\ ${ }^{a}$ Memorial Sloan-Kettering Cancer Center, New York, N.Y., and ${ }^{b}$ Department of \\ Ophthalmology, Children's Hospital Boston, Harvard Medical School, Boston, \\ Mass., USA; 'The School of Medicine, Dentistry and Biomedical Sciences, \\ Queen's University, Belfast, UK
}

\section{Key Words}

Papilledema $\cdot$ Optic atrophy $\cdot$ Rickets $\cdot$ Craniosynostosis

\begin{abstract}
Purpose: Introduction to the ophthalmic literature of an unusual cause of papilledema and subsequent optic atrophy: X-linked hypophosphatemic rickets (XLH).

Methods: Case report of a 3-year-old female presenting with papilledema resulting from craniosynostosis secondary to XLH.

Results: Early intervention with craniofacial surgery prevented the development of optic atrophy.

Conclusion: Children with XLH should be screened for ophthalmic evidence of elevated intracranial pressure to aid early intervention and prevention of permanent loss of vision.
\end{abstract}

\section{Introduction}

Papilledema is an ominous, if non-specific sign in the pediatric population, indicating elevated intracranial pressure that may derive from a variety of sources, including mass lesions, hemorrhage, hydrocephalus, craniosynostosis or idiopathic intracranial hypertension. This report describes a case of papilledema secondary to acquired craniosynostosis in a child with X-linked hypophosphatemic rickets (XLH). Patients with XLH present with evidence of rickets, such as leg bowing, within the first years of life [1]. In addition, and in common with all patients with rickets, patients with XLH may develop craniosynostosis [2]. Although the association of craniosynostosis with papilledema is well described, this highly unusual metabolic etiology of craniosynostosis and associated papilledema has received no prior recognition in the ophthalmic literature. Lack of suspicion may delay evaluation for associated papilledema; deferred intervention could result in optic atrophy. 


\section{Case Report}

A 3-year-old female child with XLH was referred to our service for evaluation of possible papilledema. Asymptomatic sagittal synostosis had been tentatively diagnosed at an outside institution on screening plane skull films. She was under treatment with calcitriol and sodium phosphate with potassium phosphate (Neutra-Phos).

Uncorrected acuity was 20/40 in each eye. She was orthophoric at distance and near, and demonstrated $200 \mathrm{~s}$ of stereopsis. Anterior segment examination, intraocular pressure, and confrontation fields were normal. Funduscopic examination revealed disc elevation and blurring of the margins, in the right eye more than in the left, highly suggestive of papilledema (fig. 1 , upper photos). Cycloplegic refraction was +6.00 diopters in the right eye and $+4.25 /+0.50 \times 135$ diopters in the left eye. Papilledema, pseudo-papilledema related to high hyperopia, and drusen were considered as possible etiologies for the fundus findings. Glasses were prescribed for her hyperopia and anisometropia. A CT scan of the orbits and brain demonstrated sagittal synostosis, metopic suture synostosis, nearly closed coronal and lambdoidal sutures, dolichocephaly, and bilaterally enlarged optic nerve sheaths consistent with papilledema. No drusen were visualized and the cerebrospinal fluid space was severely limited (fig. 2 , fig. 3 ). Thus, elevated intracranial pressure was further confirmed by radiologic imaging.

On this basis, the patient underwent biparietal widening and frontal and occipital outfracture. At the time of surgery, neurosurgical and plastics colleagues noted severe interdigitation of the brain into the bony skull with bone erosion and scalloping, and near elimination of the cerebrospinal fluid space, all consistent with elevation of intracranial pressure. As a result of their intervention, her skull circumference increased by $2 \mathrm{~cm}$ and intracranial pressure normalized. Disc edema resolved (fig. 1, lower photos). Ophthalmic care was otherwise confined to the management of unrelated anisometropic amblyopia and accommodative esotropia. Both color vision and the visual fields remained normal throughout. There was no clinical evidence of secondary optic atrophy.

Literature Search

A Medline search without limiting year or language was done linking the search terms "rickets", "papilledema", "optic atrophy", and "craniosynostosis".

\section{Discussion}

Rickets is a childhood disease in which new bone fails to mineralize [3]. It is classified as either hypocalcemic or normocalcemic. Hypocalcemic rickets can result from insufficient vitamin D due to dietary, malabsorptive, metabolic or environmental etiologies, among others. Normocalcemic rickets, in contrast, is caused by inorganic phosphate wasting in the proximal renal tubules. For this reason, it is also described as vitamin D resistant [4].

Of the vitamin D-resistant forms of rickets, XLH is the most common subtype [4]. The PHEX gene is inactivated in most XLH patients [5]. Though the exact mechanism relating PHEX inactivation to the findings in XLH is unclear, there is evidence that PHEX might regulate fibroblast growth factor 23 (FGF23) [6]. FGF23, in turn, regulates phosphate homeostasis and vitamin D metabolism, and may influence new bone mineralization [1].

Patients with XLH generally appear normal at birth, but manifest short stature, osteomalacia, bowleggedness, and rickets within the first two years of life [1]. In common with all patients with rickets, they may also develop craniosynostosis. It has 
been suggested that bridges of new bone crossing suture lines may inhibit suture mobility and result in premature fusion [2].

Thus, in a study published in 1964, Reilly et al. [7] found that 16 of 59 patients with rickets from any cause had evidence of complete fusion of at least one suture by age 9 years (mean 3.8), and estimated that at least 3 more patients could have been added had they not died in infancy. The sagittal suture was most often affected. Not surprisingly, the greater the age at initial presentation for rickets, and the milder the symptomatology, the lower the incidence and the lesser the severity of craniosynostosis. Children with vitamin D-refractory rickets (presumably including $\mathrm{XLH}$ ) often presented after the first year of life and were least affected. This study, however, preceded CT scanning, and probably underestimates the true prevalence of craniosynostosis.

Although many patients show gross signs of craniosynostosis, not all cases of craniosynostosis are clinically apparent. A recent case report [8], for example, describes an otherwise asymptomatic 1-year-old child with treated XLH, unrelated hemangiomas of the face and scalp, and bilateral papilledema. A CT scan demonstrated sagittal synostosis, and elevated intracranial pressure was documented on lumbar puncture. As in our case, the synostosis was clinically silent.

A study in 2007 [4] reviewed 28 children with hypophosphatemic rickets. Sagittal synostosis was present in 13 (46\%) of them. Ten patients were dolichocephalic, and 3 were mesocephalic nearing brachycephaly. The author of the paper posited that the 3 patients with mesocephaly were likely clinically asymptomatic due to the formation of craniosynostosis during 'a period of slower brain growth, perhaps starting late in the second year of life'.

Whereas secondary craniosynostosis may cause disc edema or optic atrophy in some children with rickets, another report [9], from 1994, implicates compressive optic atrophy from optic canal and superior orbital fissure narrowing, and bilateral globe calcification. The authors suggest that these findings may have been a function of natural history combined with longstanding vitamin D supplementation causing intoxication with subsequent metastatic calcification.

Although the constellation of craniosynostosis, elevated intracranial pressure, secondary papilledema, and optic atrophy in the pediatric population is well known, 'secondary' craniosynostosis from rickets is quite rare and has not been noted previously in the ophthalmic literature. We present this case to remind our colleagues of this rare and highly treatable etiology of elevated intracranial pressure, papilledema, and optic atrophy. Prompt referral for craniofacial surgical intervention can prevent or abate permanent vision loss.

\section{Disclosure Statement}

The research was conducted at Children's Hospital Boston and has not been presented previously; none of the authors has any financial interest in the research presented. There are no conflicts of interest to disclose. No funding was received for this work. 


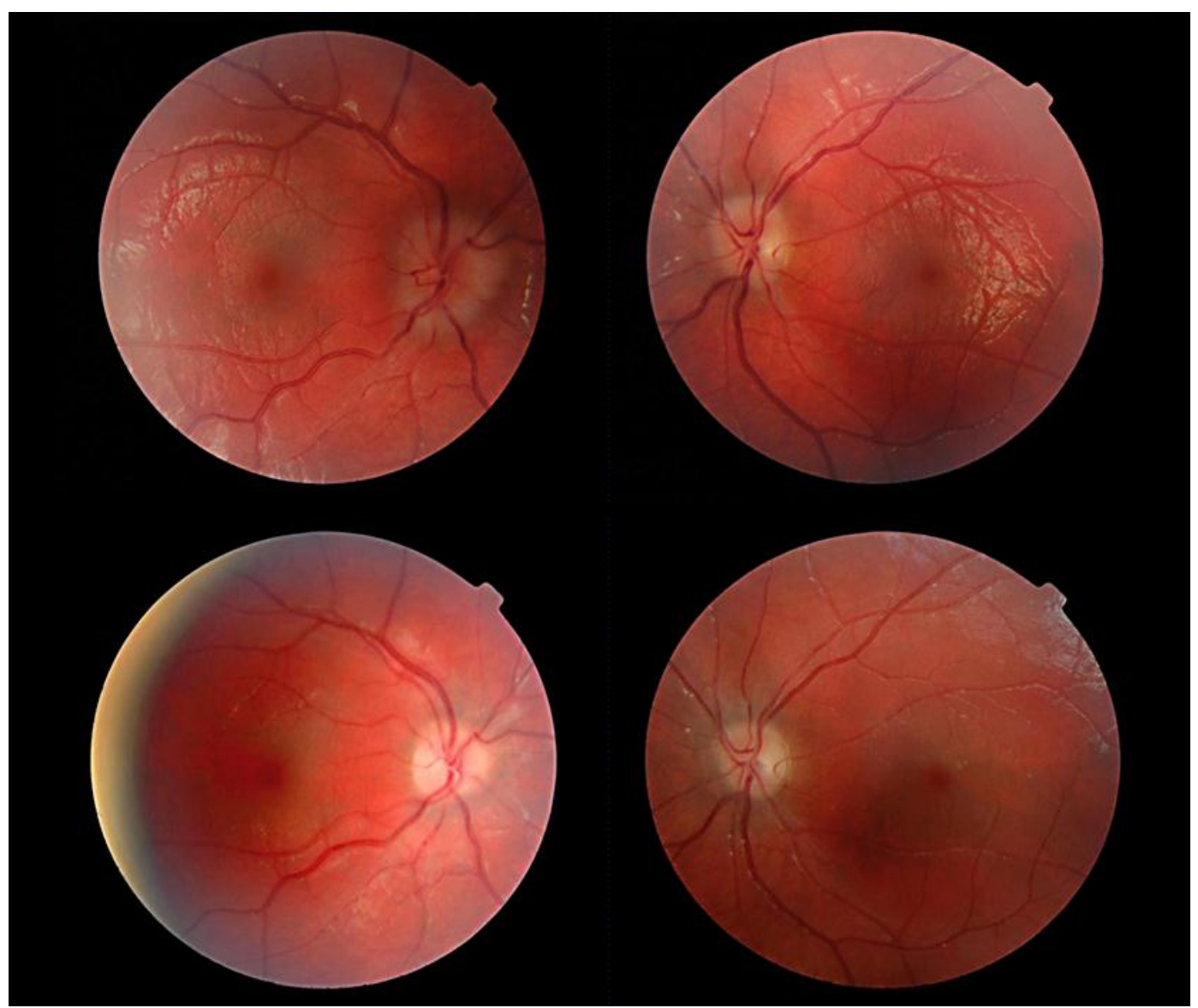

Fig. 1. Fundus photographs. The upper photographs illustrate disc edema, greater in the right eye than in the left. The lower photographs document resolution of the same disc edema after biparietal widening and frontal and occipital outfracture. 


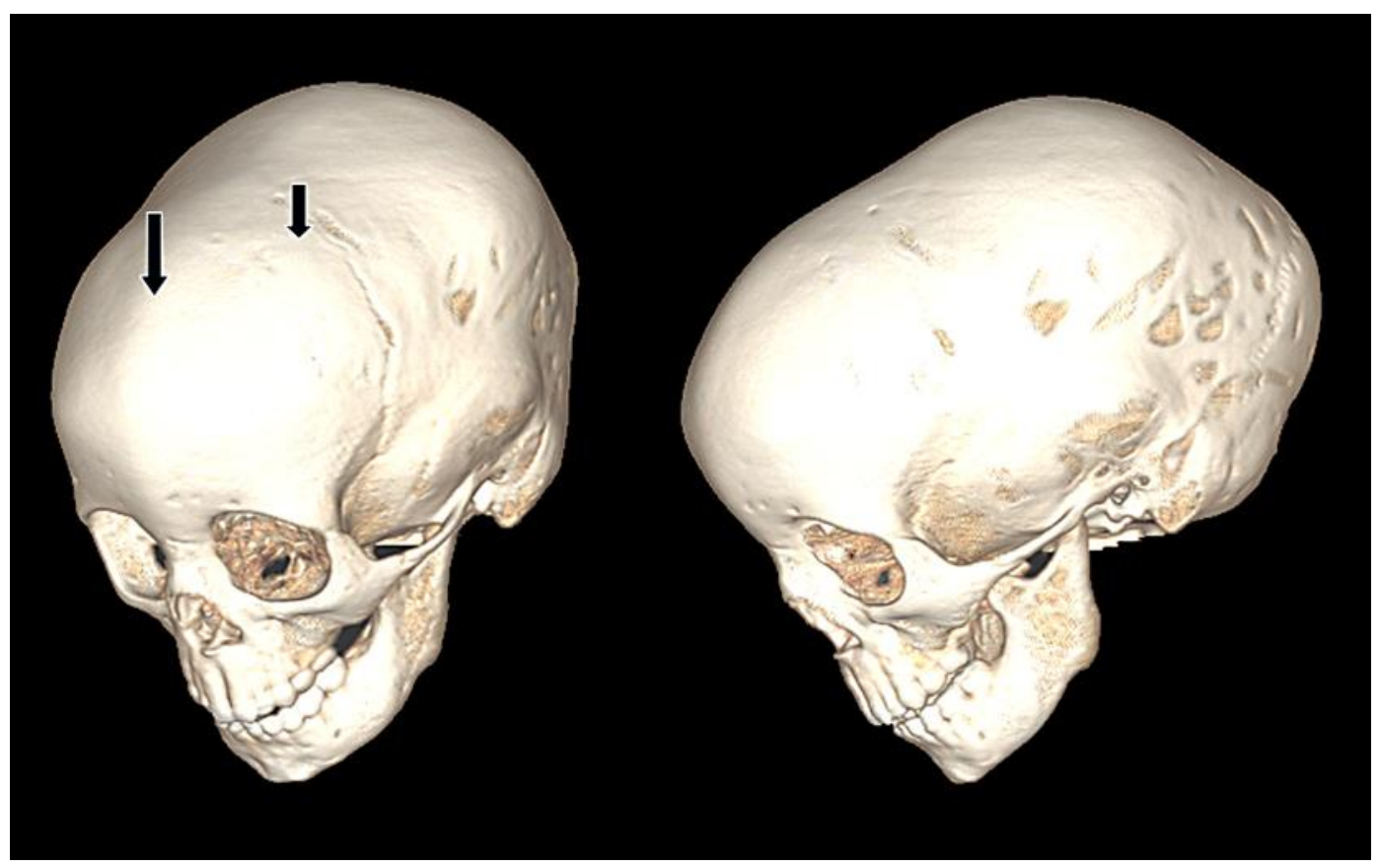

Fig. 2. CT scan. The left image documents metopic synostosis (left arrow) and partial coronal synostosis (right arrow). The right image demonstrates dolichocephaly (anterior-posterior elongation of the cranial vault).

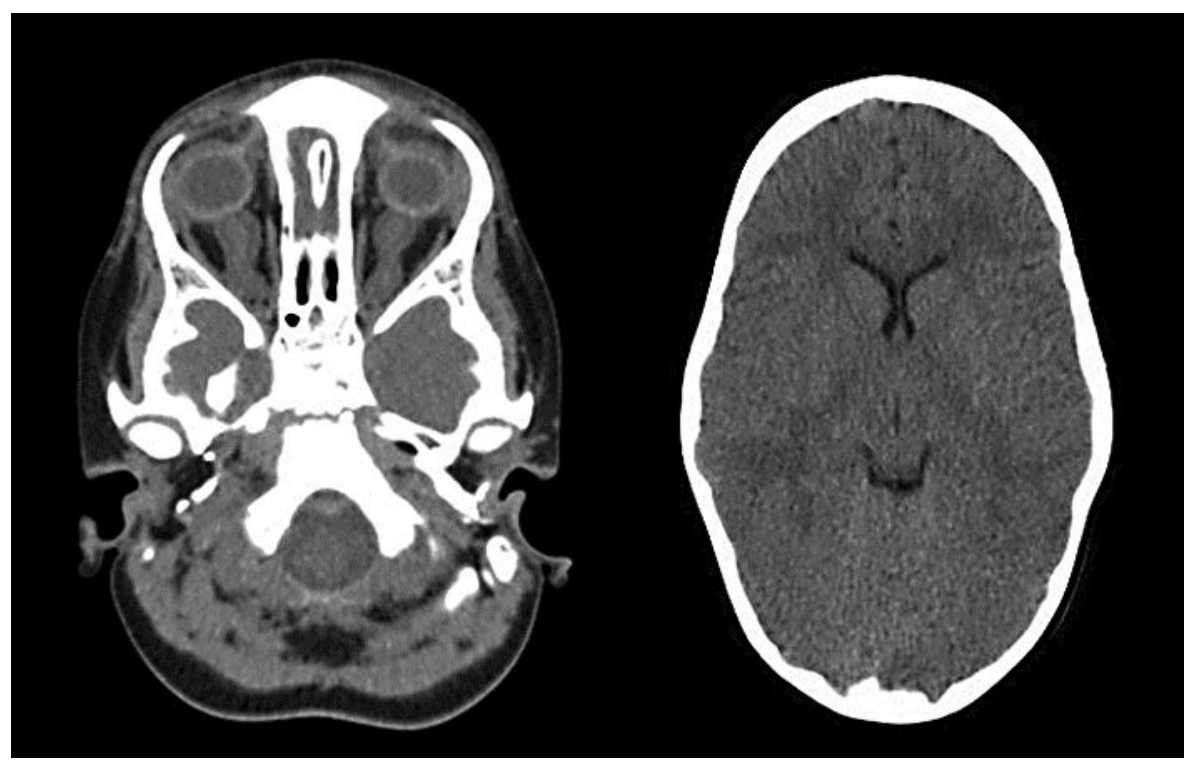

Fig. 3. Brain and orbital CT scan. The left image shows enlargement of optic nerve sheaths secondary to papilledema. The right image illustrates ventricular narrowing and near absence of cerebrospinal fluid space. 


\section{References}

1 Pettifor JM: What's new in hypophosphatemic rickets? Eur J Pediatr 2008;167:493-499.

$\longrightarrow 2$ Sitsen AE: Das Verhalten der Schädeldachnähte bei Rachitis. Osterr Z Kinderheilkd Kinderfuersorge 1948;1:375-393.

-3 McCarthy JG, Reid CA: Craniofacial synostosis in association with vitamin D-resistant rickets. Ann Plast Surg 1980;4:149-153.

4 Currarino G: Sagittal synostosis in X-linked hypophosphatemic rickets and related diseases. Pediatr Radiol 2007;37:805-812.

5 The HYP Consortium: A gene (PEX) with homologies to endopeptidases is mutated in patients with Xlinked hypophosphatemic rickets. Nature Genet 1995;11:130-136.

6 Garabedian M: Regulation of phosphate homeostasis in infants, children, and adolescents, and the role of phosphatonins in this process. Curr Opin Pediatr 2007;19:488-491.

7 Reilly BJ, Leeming JM, Fraser D: Craniosynostosis in the rachitic spectrum. J Pediatr 1964;64:396-405.

-8 Murthy AS: X-linked hypophosphatemic rickets and craniosynostosis. J Craniofac Surg 2009;20:439442.

9 Caldemeyer KS, Smith RR, Edwards-Brown MK: Familial hypophosphatemic rickets causing ocular calcification and optic canal narrowing. Am J Neuroradiol 1995;16:1252-1254. 